\title{
Prevalence of Hoarseness and Its Association with Severity of Dysphagia in Patients with Sarcopenic Dysphagia
}

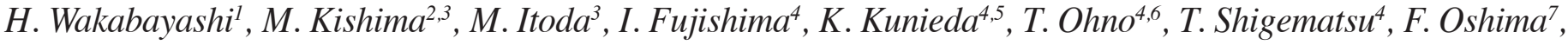 \\ T. Mori ${ }^{8}$, N. Ogawa, S. Nishioka ${ }^{10}$, R. Momosaki ${ }^{11}$, M. Yamada ${ }^{12}$, S. Ogawa ${ }^{13}$, The Japanese Working Group \\ on Sarcopenic Dysphagia
}

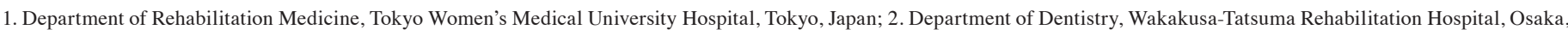

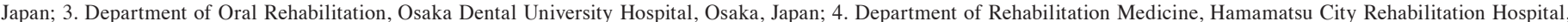

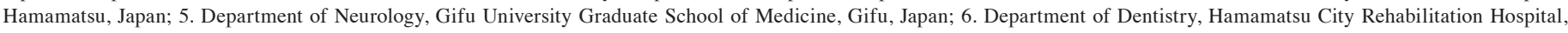

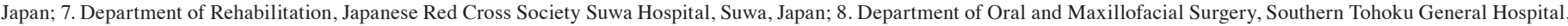

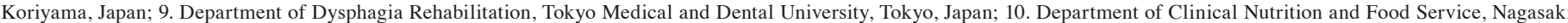

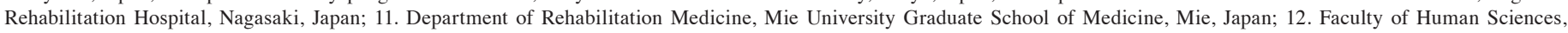
University of Tsukuba, Tokyo, Japan; 13. Department of Geriatric Medicine, Graduate School of Medicine, University of Tokyo, Bunkyo-ku, Tokyo, Japan
\end{abstract}

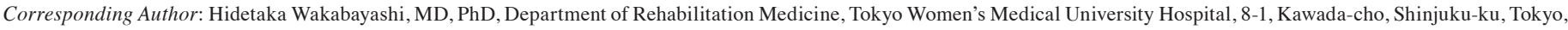
Japan. Code; 162-0054, Tel: +81-3-3353-8111, Fax: +81-3-5269-7639, E-mail: noventurenoglory@gmail.com

\begin{abstract}
OBJECTIVES: To investigate the prevalence of hoarseness and its association with the severity of dysphagia in patients with sarcopenic dysphagia.

DESIGN: Cross-sectional study using the Japanese sarcopenic dysphagia database.

SETTING: 19 hospitals including 9 acute care hospitals, 8 rehabilitation hospitals, 2 long-term care hospitals, and 1 home visit rehabilitation team.

PARTICIPANTS: 287 patients with sarcopenic dysphagia, aged 20 years and older.

Measurements: Sarcopenic dysphagia was diagnosed using a reliable and validated diagnostic algorithm for the condition. The presence and characteristics of hoarseness classified as breathy, rough, asthenic, and strained were assessed. The prevalence of hoarseness and the relationship between hoarseness and Food Intake LEVEL Scale (FILS) were examined. Order logistic regression analysis adjusted for age, sex, naso-gastric tube, and handgrip strength was used to examine the relationship between hoarseness and FILS at baseline and at follow-up. RESULTS: The mean age was $83 \pm 10$ years. Seventy-four $(26 \%)$ patients had hoarseness, while $32(11 \%), 20(7 \%), 22(8 \%)$, and 0 $(0 \%)$ patients had breathy, rough, asthenic, and strained hoarseness, respectively. Median FILS at the initial evaluation was 7 (interquartile range, 5-8). Hoarseness $(\beta=0.747,95 \%$ confidence intervals $=0.229$, $1.265, \mathrm{p}=0.005)$, age, sex, naso-gastric tube, and handgrip strength were associated independently with baseline FILS, while hoarseness $(\beta=0.213,95 \%$ confidence intervals $=-0.324,0.750, p=0.438)$ was not associated independently with the FILS at follow-up.

CONCLUSIONS: Hoarseness was associated with the severity of dysphagia at baseline, however not a prognostic factor for sarcopenic dysphagia. Resistance training of swallowing and respiratory muscles and voice training as part of rehabilitation nutrition might be useful for treating sarcopenic dysphagia.
\end{abstract}

Key words: Voice, deglutition, sarcopenia, rehabilitation, nutrition.

\section{Introduction}

arcopenic dysphagia is defined as difficulty swallowing due to sarcopenia of the generalized skeletal and swallowing muscles and is a common disorder in older people $(1,2)$. Sarcopenia of the generalized skeletal muscles has been associated with many adverse health consequences, not limiting to dysphagia (3). A systematic review and meta-analysis showed that whole body sarcopenia was associated independently with dysphagia (4). Another systematic review and meta-analysis reported that reduced tongue strength was associated with sarcopenia but not with sarcopenic dysphagia (5). However, reduced tongue strength is associated with malnutrition (6). A study in a mouse model indicated that aspiration pneumonia, a common cause of sarcopenic dysphagia, induced muscle atrophy not only in skeletal muscle but also in the tongue and diaphragm (7). Sarcopenic dysphagia may also occur after coronavirus disease 2019 (COVID-19) (8), with lower handgrip strength reported to be associated with hospitalization due to COVID-19 (9). Moreover, the prevalence of sarcopenic dysphagia in inpatients who required dysphagia rehabilitation, inpatients with pneumonia, and institutionalized older people was $32 \%, 81 \%$, and $45 \%$, respectively (10-12). Therefore, sarcopenic dysphagia represents a very important issue in geriatric nutrition in both clinical and research settings.

Hoarseness and dysphagia can occur simultaneously, although the relationship between these two symptoms in sarcopenic dysphagia is unknown. The mechanism of hoarseness is muscle tone-related irregularity in the oscillation of the vocal cords. Thyroarytenoid muscle, arytenoid muscle, lateral cricoarytenoid muscle are muscles that close glottis and support both voice and swallowing for preventing aspiration. Common causes of hoarseness are acute and chronic laryngitis, functional vocal disturbances, tumors, and vocal cord paresis (13). Both hoarseness and dysphagia occur in patients with tumors and vocal cord paresis, while patients with sarcopenic 
dysphagia can present with hoarseness as a complication (14). Sarcopenia may occur in vocal muscles because the thyroarytenoid and tongue muscles have similar histological organization (15), and also in tongue muscle (16-18). Sarcopenia in vocal muscles can lead to vocal quality change. However, as far as we are aware the prevalence of hoarseness and the relationship between hoarseness and swallowing function in sarcopenic dysphagia has not been studied.

The objective of this study was therefore to investigate the prevalence of hoarseness and its association with the severity of dysphagia in patients with sarcopenic dysphagia.

\section{Methods}

\section{Study design}

A cohort study was performed using the Japanese sarcopenic dysphagia database, the details of which have been reported previously (19). In summary, the Rehabilitation Nutrition Database Committee of the Japanese Association of Rehabilitation Nutrition and the Japanese Working Group on Sarcopenic Dysphagia constructed the database. Dysphagic patients aged 20 years and older, with a Food Intake LEVEL Scale (FILS) $(20) \leq 8$ were included in the database. FILS levels 1-3 relate to various degrees of non-oral feeding, levels 4-6 to various degrees of oral food intake and alternative nutrition, levels 7-8 to various degrees of oral food intake alone, level 9 to no dietary restriction but medical consideration given, while level 10 indicates normal oral food intake. All settings such as acute care, rehabilitation or long-term care hospitals, other facilities, and home visit rehabilitation were included in the study. Baseline and follow-up data were collected, with follow-up data recorded for hospitalized patients at the time of discharge. Follow-up data were collected after 3 months of patients living at home and those who had been hospitalized for longer than 3 months since the baseline assessment.

\section{Participant}

The inclusion criterion was registration in the database, while the exclusion criterion was not having sarcopenic dysphagia. Patients aged less than 65 years were included, because only $13(4.5 \%)$ patients with sarcopenic dysphagia were less than 65 years (19). Sarcopenic dysphagia was diagnosed using a reliable and validated diagnostic algorithm for the disorder (21-23). This diagnostic algorithm divides patients into three categories: probable, possible, or no sarcopenic dysphagia. The presence of whole body sarcopenia diagnosed by the Asian Working Group for Sarcopenia (AWGS) 2019 criteria (24), the presence of dysphagia, and no other causative diseases of dysphagia other than sarcopenia were necessary for the diagnosis of sarcopenic dysphagia. Patients with low swallowing muscle strength assessed by tongue pressure measurements were diagnosed as probable sarcopenic dysphagia, while people with normal swallowing muscle strength or those in whom it was not possible to measure swallowing muscle strength were diagnosed as possible sarcopenic dysphagia. The ethics committee of the Yokohama City University Medical Center approved the study (B190700074). All participants provided informed consent prior to enrollment or were given the right to refuse participation in the study (i.e., opt out option).

\section{Variables}

The presence and characteristics of hoarseness such as breathy, rough, asthenic, and strained were assessed as same as the GRBAS scale (25). However, the severity of hoarseness was not evaluated unlike the GRBAS scale (25). The Barthel Index (26), updated Charlson comorbidity index (27), FILS, calf circumference, handgrip strength, maximum tongue pressure, body mass index, malnutrition diagnosed by the Global Leadership Initiative on Malnutrition (GLIM) criteria (28), C-reactive protein level, serum albumin level, and route of nutritional administration such as oral intake, naso-gastric tube, gastrostomy, total parenteral nutrition, or peripheral parenteral nutrition were also evaluated. FILS and the Barthel Index were assessed at follow-up.

\section{Statistical method}

Statistical analysis of the data was performed using the IBM Statistical Package for the Social Sciences (SPSS) ver. 26 software (IBM Corporation; Armonk, New York, US). Parametric data were expressed as the mean \pm standard deviation (SD) and nonparametric data as the median and interquartile range (IQR). The Chi-square test, Mann-Whitney $\mathrm{U}$ test, and t-test were used to analyze differences in the presence or absence of hoarseness. The Kruskal-Wallis test was used to analyze the differences in FILS between types of hoarseness. Order logistic regression analysis adjusted for age, sex, naso-gastric tube, and handgrip strength was conducted to examine the relationship between hoarseness and FILS at baseline, because naso-gastric tube can affect hoarseness more than other routes of nutritional administration. To determine whether hoarseness was a prognostic factor for dysphagia, order logistic regression analysis adjusted for age, sex, naso-gastric tube, handgrip strength, and FILS at baseline was performed. Tongue pressure should also have been adjusted, although there were many missing values $(\mathrm{N}=79)$ in the study, because tongue pressure measurement instrument (JMS Hiroshima, Japan) was not available in several hospitals. Therefore, handgrip strength was adjusted instead, as it is known to be associated with both swallowing function (29) and tongue pressure (30). A P value $<0.05$ was considered statistically significant.

\section{Results}

The number of registered patients in the Japanese sarcopenic dysphagia database was 467 . Of these 467 registered patients, $180(39 \%)$ patients who were not diagnosed as sarcopenic dysphagia were excluded. The remaining 287 (61\%) patients (127 males and 180 females, mean age \pm standard deviation, 
Table 1. The baseline demographic data

\begin{tabular}{|c|c|c|c|c|}
\hline & Total $\mathrm{N}=\mathbf{2 8 7}$ & Hoarseness (+) $\mathrm{N}=74$ & Hoarseness (-) $N=213$ & P-value \\
\hline Age, years, mean \pm SD & $83 \pm 10$ & $82 \pm 9$ & $83 \pm 10$ & $0.231^{\mathrm{a}}$ \\
\hline Sex, n (\%) & & & & $<0.001^{\mathrm{b}}$ \\
\hline Female & $160(56 \%)$ & $23(31 \%)$ & $137(64 \%)$ & \\
\hline Barthel Index, median (IQR) & $25(5,50)$ & $20(0,45)$ & $30(5,50)$ & $0.204^{\mathrm{c}}$ \\
\hline 1 & $28(10 \%)$ & $11(15 \%)$ & $17(8 \%)$ & \\
\hline 2 & $24(8 \%)$ & $11(15 \%)$ & $13(6 \%)$ & \\
\hline 3 & $11(4 \%)$ & $3(4 \%)$ & $8(4 \%)$ & \\
\hline 4 & $3(1 \%)$ & $0(0 \%)$ & $3(1 \%)$ & \\
\hline 8 & $89(31 \%)$ & $11(15 \%)$ & $78(37 \%)$ & \\
\hline Calf circumference $(\mathrm{cm})$, mean $\pm \mathrm{SD}$ & $27.2 \pm 3.8$ & $27.2 \pm 4.0$ & $27.2 \pm 3.8$ & $0.987^{\mathrm{a}}$ \\
\hline Handgrip strength $(\mathrm{kg})$, mean $\pm \mathrm{SD}$ & $10.9 \pm 7.2$ & $12.2 \pm 8.9$ & $10.4 \pm 6.4$ & $0.114^{\mathrm{a}}$ \\
\hline Tongue pressure $(\mathrm{kPa})$, mean $\pm \mathrm{SD}$ & $15.4 \pm 10.8$ & $13.3 \pm 10.1$ & $16.1 \pm 11.0$ & $0.312^{\mathrm{a}}$ \\
\hline Possible sarcopenic dysphagia, $\mathrm{n}(\%)$ & $182(63 \%)$ & $46(62 \%)$ & $136(64 \%)$ & $0.795^{\mathrm{b}}$ \\
\hline Probable sarcopenic dysphagia, n (\%) & $105(37 \%)$ & $28(38 \%)$ & $77(36 \%)$ & \\
\hline Body mass index $\left(\mathrm{kg} / \mathrm{m}^{2}\right)$, mean $\pm \mathrm{SD}$ & $19.6 \pm 5.1$ & $19.4 \pm 3.5$ & $19.7 \pm 3.6$ & $0.488 \mathrm{a}^{\mathrm{a}}$ \\
\hline GLIM Malnutrition, $\mathrm{n}(\%)$ & $209(73 \%)$ & $57(77 \%)$ & $152(71 \%)$ & $0.638^{\mathrm{b}}$ \\
\hline C-reactive protein $(\mathrm{mg} / \mathrm{dL})$, median $(\mathrm{IQR})$ & $0.9(0.3,4.0)$ & $1.5(0.3,5.2)$ & $0.8(0.3,3.0)$ & $0.275^{\mathrm{c}}$ \\
\hline Total parenteral nutrition & $5(2 \%)$ & $1(1 \%)$ & $4(2 \%)$ & $0.017^{\mathrm{b}}$ \\
\hline Peripheral parenteral nutrition & $43(15 \%)$ & $24(32 \%)$ & $19(9 \%)$ & $0.003^{\mathrm{b}}$ \\
\hline
\end{tabular}

a: t-test, b: Chi-square test, c: Mann-Whitney U test; GLIM: Global Leadership Initiative on Malnutrition, IQR: interquartile range, SD: standard deviation

$83 \pm 10$ years), participated in the cohort study (Figure 1). The number of patients with probable sarcopenic dysphagia was 105 (37\%) and possible sarcopenic dysphagia $182(63 \%)$.

Table 1 shows the comparative tests for differences between patients with or without hoarseness at baseline. Seventy-four (26\%) patients had hoarseness, with males significantly more likely to have hoarseness than females. FILS at baseline was significantly lower in the hoarseness group. However, there was no significant difference in the incidence of possible sarcopenic dysphagia and probable sarcopenic dysphagia between the two groups. Naso-gastric tube feeding was significantly more common in the group with hoarseness, whereas total and peripheral parenteral nutrition were significantly more common in the group without hoarseness. Oral intake was more common in the group without hoarseness group, although this difference was not statistically significant.
Figure 1. Flowchart of participants

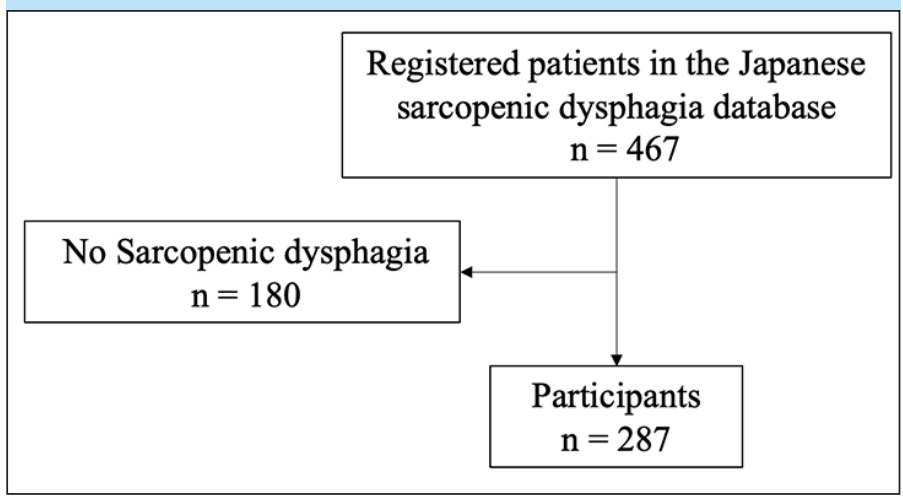

Of 467 registered patients, 287 patients with sarcopenic dysphagia participated in the cohort study. 
Table 2. The follow-up demographic data

\begin{tabular}{|c|c|c|c|c|}
\hline & Total & Hoarseness (+) & Hoarseness (-) & P-value \\
\hline Barthel Index, median (IQR) & $25(5,50)$ & $42.5(15,68.75)$ & $60(20,80)$ & $0.057^{\mathrm{a}}$ \\
\hline Food Intake LEVEL Scale, n (\%) & & & & $0.023^{\mathrm{a}}$ \\
\hline 1 & $4(1 \%)$ & $1(1 \%)$ & $3(2 \%)$ & \\
\hline 2 & $16(6 \%)$ & $4(6 \%)$ & $12(6 \%)$ & \\
\hline 3 & $4(1 \%)$ & $1(1 \%)$ & $3(2 \%)$ & \\
\hline 4 & $5(2 \%)$ & $3(4 \%)$ & $2(1 \%)$ & \\
\hline 5 & $3(1 \%)$ & $2(3 \%)$ & $1(1 \%)$ & \\
\hline 6 & $13(5 \%)$ & $7(10 \%)$ & $6(3 \%)$ & \\
\hline 7 & $81(30 \%)$ & $23(34 \%)$ & $58(29 \%)$ & \\
\hline 8 & $103(38 \%)$ & $17(25 \%)$ & $86(43 \%)$ & \\
\hline 9 & $24(9 \%)$ & $8(12 \%)$ & $16(8 \%)$ & \\
\hline 10 & $15(6 \%)$ & $2(3 \%)$ & $13(7 \%)$ & \\
\hline Outcome including discharge destination & & & & $0.411^{\mathrm{b}}$ \\
\hline Discharge to home & $153(57 \%)$ & $38(51 \%)$ & $115(54 \%)$ & \\
\hline Transfer to other hospitals/facilities & $92(34 \%)$ & $24(32 \%)$ & $68(32 \%)$ & \\
\hline Continuation of hospitalization or home life & $23(9 \%)$ & $6(8 \%)$ & $17(8 \%)$ & \\
\hline Death & $19(7 \%)$ & $6(8 \%)$ & $13(6 \%)$ & \\
\hline
\end{tabular}

a: Mann-Whitney U test, b: Chi-square test; IQR: interquartile range

Table 2 shows the comparative tests for differences between patients with or without hoarseness at follow-up. There were no follow-up data on the Barthel index or FILS for 19 patients who had died. The Barthel index was higher in the group without hoarseness, although this difference was not statistically significant. FILS at follow-up was significantly lower in the group with hoarseness. There was no significant difference in outcomes including the destination of discharge between the two groups.

Table 3 shows the relationship between the type of hoarseness and FILS. 32 (11\%), 20 (7\%), $22(8 \%)$, and $0(0 \%)$ patients had breathy, rough, asthenic, and strained hoarseness, respectively. There were no significant differences in FILS at baseline and follow-up in patients with breathy, rough, and asthenic hoarseness.

Table 4 shows ordered logistic regression analysis of the FILS at baseline and follow-up adjusted for the presence of hoarseness, age, sex, and handgrip strength. Hoarseness $(\beta=0.747,95 \%$ confidence intervals $=0.229,1.265, p=0.005)$, age, sex, naso-gastric tube, and handgrip strength were associated independently with baseline FILS, while hoarseness $(\beta=0.213,95 \%$ confidence intervals $=-0.324,0.750, p=0.438)$ was not associated independently with the FILS at followup. Handgrip strength and baseline FILS were associated independently with FILS at follow-up.

\section{Discussion}

This is the first study to examine the prevalence of hoarseness and its relationship with the severity of dysphagia in patients with sarcopenic dysphagia. The prevalence of hoarseness was $26 \%$ in these patients. Hoarseness was associated with the severity of dysphagia at baseline. However, hoarseness was not a prognostic factor for sarcopenic dysphagia. The severity of dysphagia was not different in patients with different types of hoarseness.

Table 3. Relationship between type of hoarseness and swallowing function

\begin{tabular}{|l|c|c|}
\hline Type of hoarseness & Food Intake LEVEL Scale & P-value \\
\hline $\begin{array}{l}\text { At baseline, median (IQR) } \\
\text { breathy }\end{array}$ & $7(4.25,7)$ & 0.220 \\
rough & $6(2,7)$ & \\
asthenic & $7(1.5,7)$ & \\
At follow-up, median (IQR) & $7(7,8.25)$ & 0.170 \\
breathy & $7(5,8)$ & \\
rough & $7(6,7)$ & \\
asthenic & & \\
\hline
\end{tabular}

IQR: interquartile range

Nearly one-fourth of patients with sarcopenic dysphagia had hoarseness. The prevalence of hoarseness in general dysphagia including sarcopenic dysphagia was $31 \%$ according to the Japanese Sarcopenic Dysphagia Database (19). The prevalence of hoarseness was higher in patients with dysphagia due to stroke and neurodegenerative diseases, because these diseases can cause hoarseness due to vocal cord paralysis. However, hoarseness is common in patients with sarcopenic dysphagia. There are several mechanisms for hoarseness in sarcopenic dysphagia, although the control style of swallowing-related muscles and speech-related muscles is different at the central 
Table 4. Ordered logistic regression analysis at baseline and follow-up

\begin{tabular}{|c|c|c|c|c|}
\hline & B & \multicolumn{2}{|c|}{ 95\% Confidence Interval } & P-value \\
\hline \multicolumn{5}{|l|}{ At baseline } \\
\hline Age & 0.038 & 0.002 & 0.014 & 0.002 \\
\hline Sex & -0.871 & -1.357 & -0.385 & $<0.001$ \\
\hline Naso-gastric tube & 2.523 & 1.820 & 3.227 & $<0.001$ \\
\hline Handgrip strength & 0.065 & 0.032 & 0.098 & $<0.001$ \\
\hline Hoarseness & 0.747 & 0.229 & 1.265 & 0.005 \\
\hline \multicolumn{5}{|l|}{ At follow-up } \\
\hline Age & -0.014 & -0.039 & 0.010 & 0.238 \\
\hline Sex & -0.290 & -0.803 & 0.223 & 0.267 \\
\hline Naso-gastric tube & 0.752 & -0.027 & 1.530 & 0.058 \\
\hline Handgrip strength & 0.063 & 0.028 & 0.097 & $<0.001$ \\
\hline Hoarseness & 0.213 & -0.324 & 0.750 & 0.438 \\
\hline Food Intake LEVEL Scale at baseline & 0.357 & 0.228 & 0.485 & $<0.001$ \\
\hline
\end{tabular}

pattern generator level, and the contribution of each muscle is different. Sarcopenia of the thyroarytenoid muscle can occur and result in hoarseness in patients with severe whole body sarcopenia and sarcopenic dysphagia. Respiratory sarcopenia and sarcopenic respiratory disability (31) may also induce hoarseness as a consequence of expiratory impairment, while gastroesophageal and laryngopharyngeal reflux are known to complicate dysphagia in patients. In addition, acute and chronic laryngitis are more likely to occur in patients with dysphagia because of aspiration and may result in the development of hoarseness. Taken together, these findings indicate that the voice should be assessed in patients with sarcopenic dysphagia and that the mechanism of hoarseness needs to be studied in greater detail.

Hoarseness was associated with the severity of dysphagia at baseline, however not a prognostic factor for sarcopenic dysphagia. Whole body sarcopenia is naturally a risk factor for sarcopenic dysphagia $(32,33)$, while activities of daily living, malnutrition, insufficient nutritional intake, and length of stay have been reported to increase the risk of developing sarcopenic dysphagia (34). Our study showed that handgrip strength included in whole body sarcopenia diagnosis was a prognostic factor for sarcopenic dysphagia. Hoarseness could not be used to predict prognosis of sarcopenic dysphagia. We found that the severity of dysphagia was not different with breathy, rough, or asthenic hoarseness, although the mechanism of hoarseness may have been different in these patient groups. At least, strained hoarseness appeared not to occur in patients with sarcopenic dysphagia.

Dysphagia rehabilitation including voice training and expiratory muscle strength training may be useful for patients with both hoarseness and sarcopenic dysphagia. There is evidence that resistance training of the suprahyoid muscles improved not only swallowing function but also vocal function in healthy older adults $(35,36)$. Voice therapy was also reported to improve not only voice function but also swallowing function in patients with either Parkinson's disease, multiple system atrophy, or progressive supranuclear palsy (37-40).
Moreover, expiratory muscle strength training was effective at strengthening the geniohyoid muscle (41). Interdisciplinary rehabilitation nutrition may therefore be useful for treating patients with sarcopenic dysphagia $(23,42)$ and it may be more useful to implement both resistance training of swallowing and respiratory muscles and voice training as part of rehabilitation nutrition.

There were some limitations in this study. First, we did not assess the severity of hoarseness unlike the GRBAS scale (25). Second, we did not perform laryngoscopy and videoendoscopic evaluation of swallowing and it is possible some patients with hoarseness may have been complicated by vocal cord palsy.

In conclusion, the prevalence of hoarseness in our patient cohort was $26 \%$. The prevalence of hoarseness was lower compared with general dysphagia. Hoarseness was associated with the severity of dysphagia at baseline, however not a prognostic factor for sarcopenic dysphagia. The combination of resistance training of swallowing and respiratory muscles and voice training as part of rehabilitation nutrition might be useful for treating sarcopenic dysphagia.

Disclosure statement: Hidetaka Wakabayashi reports grants from JSPS KAKENHI Grant Number 19H03979 during conduct of the study.

Conflict of interest: Hidetaka Wakabayashi, Masako Kishima, Masataka Itoda, Ichiro Fujishima, Kenjiro Kunieda, Tomohisa Ohno, Takashi Shigematsu, Fumiko Oshima, Takashi Mori, Nami Ogawa, Shinta Nishioka, Ryo Momosaki, Minoru Yamada, Sumito Ogawa have nothing to disclose.

Ethical Standards: This study has been performed in accordance with the ethical standards established in the 1964 Declaration of Helsinki and later amendments.

\section{References}

1. Wakabayashi H. Presbyphagia and Sarcopenic Dysphagia: Association between Aging, Sarcopenia, and Deglutition Disorders. J Frailty Aging. 2014;3:97-103. doi:10.14283/ jfa. 2014.8

2. Nagano A, Nishioka S, Wakabayashi H. Rehabilitation Nutrition for Iatrogenic Sarcopenia and Sarcopenic Dysphagia. J Nutr Health Aging. 2019;23:256-65. doi:10.1007/s12603-018-1150-1

3. Han DS, Wu WT, Hsu PC, Chang HC, Huang KC, Chang KV. Sarcopenia Is Associated With Increased Risks of Rotator Cuff Tendon Diseases Among Community-Dwelling Elders: A Cross-Sectional Quantitative Ultrasound Study. Front Med (Lausanne). 2021;8:630009. doi: 10.3389/fmed.2021.630009. 
4. Zhao WT, Yang M, Wu HM, Yang L, Zhang XM, Huang Y. Systematic Review and Meta-Analysis of the Association between Sarcopenia and Dysphagia. J Nutr Health Aging. 2018;22:1003-9. doi:10.1007/s12603-018-1055-Z

5. Chen KC, Lee TM, Wu WT, Wang TG, Han DS, Chang KV. Assessment of Tongue Strength in Sarcopenia and Sarcopenic Dysphagia: A Systematic Review and MetaAnalysis. Front Nutr. 2021;8:684840. doi:10.3389/fnut.2021.684840

6. Chang KV, Wu WT, Chen LR, Wang HI, Wang TG, Han DS. Suboptimal Tongue Pressure Is Associated with Risk of Malnutrition in Community-Dwelling Older Individuals. Nutrients. 2021;13:1821. doi: 10.3390/nu13061821.

7. Komatsu R, Okazaki T, Ebihara S, Kobayashi M, Tsukita Y, Nihei M, et al. Aspiration pneumonia induces muscle atrophy in the respiratory, skeletal, and swallowing systems. J Cachexia Sarcopenia Muscle. 2018;9:643-53. doi:10.1002/jcsm.12297

8. Can B, İsmagulova N, Enver N, Tufan A, Cinel İ. Sarcopenic dysphagia following COVID-19 infection: A new danger. Nutr Clin Pract. 2021;36:828-32. doi:10.1002/ ncp.10731

9. Cheval B, Sieber S, Maltagliati S, Millet GP, Formánek T, Chalabaev A, et al. Muscle strength is associated with COVID-19 hospitalization in adults 50 years of age or older. J Cachexia Sarcopenia Muscle. 2021;12:1136-43. doi:10.1002/jcsm.12738

10. Wakabayashi H, Takahashi R, Murakami T. The Prevalence and Prognosis of Sarcopenic Dysphagia in Patients Who Require Dysphagia Rehabilitation. J Nutr Health Aging. 2019;23:84-8. doi:10.1007/s12603-018-1117-2

11. Miyauchi N, Nakamura M, Nakamura I, Momosaki R. Effect of early versus delayed mobilization by physical therapists on oral intake in patients with sarcopenic dysphagia after pneumonia. European Geriatric Medicine. 2019;10:603-7. doi:10.1007/s41999-019-00169-1

12. Moncayo-Hernández BA, Herrera-Guerrero JA, Vinazco S, Ocampo-Chaparro JM, Reyes-Ortiz CA. Sarcopenic dysphagia in institutionalised older adults. Endocrinol Diabetes Nutr. 2021;68:602-11. doi:10.1016/j.endinu.2021.02.010

13. Reiter R, Hoffmann TK, Pickhard A, Brosch S. Hoarseness-causes and treatments. Dtsch Arztebl Int. 2015;112:329-37. doi:10.3238/arztebl.2015.0329

14. Wakabayashi H, Uwano R. Rehabilitation Nutrition for Possible Sarcopenic Dysphagia After Lung Cancer Surgery: A Case Report. Am J Phys Med Rehabil. 2016;95:e84-9. doi: 10.1097/phm.0000000000000458

15. de Campos D, do Nascimento PS, Ellwanger JH, Gehlen G, Rodrigues MF, Jotz GP, et al. Histological organization is similar in human vocal muscle and tongue--a study of muscles and nerves. J Voice. 2012;26:811.e19-26. doi:10.1016/j.jvoice.2011.12.006

16. Borda MG, Bani Hassan E, Weon J, Wakabayashi H, Tovar-Rios DA, Oppedal K, et al. Muscle volume and intramuscular fat of the tongue evaluated with MRI predict malnutrition in people living with dementia: a five-year follow-up study. J Gerontol A Biol Sci Med Sci. 2022;77:228-234;doi:10.1093/gerona/glab224

17. Nakao Y, Yamashita T, Honda K, Katsuura T, Hama Y, Nakamura Y, et al. Association Among Age-Related Tongue Muscle Abnormality, Tongue Pressure, and Presbyphagia: A 3D MRI Study. Dysphagia. 2021;36:483-91. doi:10.1007/s00455-020-10165-4

18. Ogawa N, Mori T, Fujishima I, Wakabayashi H, Itoda M, Kunieda K, et al Ultrasonography to Measure Swallowing Muscle Mass and Quality in Older Patients With Sarcopenic Dysphagia. J Am Med Dir Assoc. 2018;19:516-22. doi:10.1016/j. jamda.2017.11.007

19. Mizuno S, Wakabayashi H, Fujishima I, Kishima M, Itoda M, Yamakawa M, et al. Construction and Quality Evaluation of the Japanese Sarcopenic Dysphagia Database. J Nutr Health Aging. 2021;25:926-932. doi:10.1007/s12603-021-1646-y

20. Kunieda K, Ohno T, Fujishima I, Hojo K, Morita T. Reliability and validity of a too to measure the severity of dysphagia: the Food Intake LEVEL Scale. J Pain Symptom Manage. 2013;46:201-6. doi:10.1016/j.jpainsymman.2012.07.020

21. Mori T, Fujishima I, Wakabayashi H, Oshima F, Itoda M, Kunieda K, et al Development, reliability, and validity of a diagnostic algorithm for sarcopenic dysphagia. JCSM Clinical Reports. 2017;2:1-10. doi:10.17987/jcsm-cr.v2i2.17

22. Fujishima I, Fujiu-Kurachi M, Arai H, Hyodo M, Kagaya H, Maeda K, et al Sarcopenia and dysphagia: Position paper by four professional organizations. Geriatr Gerontol Int. 2019;19:91-7. doi:10.1111/ggi.13591

23. Wakabayashi H, Kishima M, Itoda M, Fujishima I, Kunieda K, Ohno T, et al Diagnosis and Treatment of Sarcopenic Dysphagia: A Scoping Review. Dysphagia. 2021;36:523-31. doi:10.1007/s00455-021-10266-8
24. Chen LK, Woo J, Assantachai P, Auyeung TW, Chou MY, Iijima K, et al. Asian Working Group for Sarcopenia: 2019 Consensus Update on Sarcopenia Diagnosis and Treatment. J Am Med Dir Assoc. 2020;21:300-7.e2. doi:10.1016/j.jamda.2019.12.012

25. De Bodt MS, Wuyts FL, Van de Heyning PH, Croux C. Test-retest study of the GRBAS scale: influence of experience and professional background on perceptual rating of voice quality. J Voice. 1997;11:74-80. doi:10.1016/s0892-1997(97)80026-4

26. Mahoney FI, Barthel DW. Functional evaluation: The Barthel Index. Md State Med J. 1965;14:61-5.

27. Quan H, Li B, Couris CM, Fushimi K, Graham P, Hider P, et al. Updating and validating the Charlson comorbidity index and score for risk adjustment in hospital discharge abstracts using data from 6 countries. Am J Epidemiol. 2011;173:676-82. doi:10.1093/aje/kwq433

28. Cederholm T, Jensen GL, Correia M, Gonzalez MC, Fukushima R, Higashiguchi T, et al. GLIM criteria for the diagnosis of malnutrition - A consensus report from the global clinical nutrition community. J Cachexia Sarcopenia Muscle. 2019;10:207-17. doi: $10.1002 /$ jcsm. 12383

29. Hathaway B, Baumann B, Byers S, Wasserman-Wincko T, Badhwar V, Johnson J. Handgrip strength and dysphagia assessment following cardiac surgery. Laryngoscope. 2015;125:2330-2. doi:10.1002/lary.25175

30. Mihara Y, Matsuda KI, Ikebe K, Hatta K, Fukutake M, Enoki K, et al. Association of handgrip strength with various oral functions in 82- to 84-year-old communitydwelling Japanese. Gerodontology. 2018; doi:10.1111/ger.12341

31. Nagano A, Wakabayashi H, Maeda K, Kokura Y, Miyazaki S, Mori T, et al Respiratory Sarcopenia and Sarcopenic Respiratory Disability: Concepts, Diagnosis, and Treatment. J Nutr Health Aging. 2021;25:507-15. doi:10.1007/s12603-021-1587-5

32. Maeda K, Akagi J. Sarcopenia is an independent risk factor of dysphagia in hospitalized older people. Geriatr Gerontol Int. 2016;16:515-21. doi:10.1111/ggi.12486

33. Cha S, Kim WS, Kim KW, Han JW, Jang HC, Lim S, et al. Sarcopenia is an Independent Risk Factor for Dysphagia in Community-Dwelling Older Adults. Dysphagia. 2019;34:692-7. doi:10.1007/s00455-018-09973-6

34. Maeda K, Ishida Y, Nonogaki T, Shimizu A, Yamanaka Y, Matsuyama R, et al. Development and Predictors of Sarcopenic Dysphagia during Hospitalization of Older Adults. Nutrients. 2019;12:70. doi:10.3390/nu12010070

35. Easterling C. Does an exercise aimed at improving swallow function have an effect on vocal function in the healthy elderly? Dysphagia. 2008;23:317-26. doi:10.1007/ s00455-008-9158-Z

36. Fujiki RB, Oliver AJ, Sivasankar MP, Craig BA, Malandraki GA. Secondary Voice Outcomes of a Randomized Clinical Trial Comparing Two Head/Neck Strengthening Exercises in Healthy Older Adults: A Preliminary Report. J Speech Lang Hear Res. 2019;62:318-23. doi:10.1044/2018_jslhr-s-18-0338

37. Miles A, Jardine M, Johnston F, de Lisle M, Friary P, Allen J. Effect of Lee Silverman Voice Treatment (LSVT LOUD®) on swallowing and cough in Parkinson's disease: A pilot study. J Neurol Sci. 2017;383:180-7. doi:10.1016/j.jns.2017.11.015

38. Park A, Jang SJ, Kim NE, Kim TH, Sohn YH, Kim H, et al. Swallowing Outcomes Following Voice Therapy in Multiple System Atrophy with Dysphagia: Comparison of Treatment Efficacy with Parkinson's Disease. Dysphagia. 2022;37:198-206. doi:10.1007/s00455-021-10265-9

39. Nozaki S, Fujiu-Kurachi M, Tanimura T, Ishizuka K, Miyata E, Sugishita S, et al. Effects of Lee Silverman Voice Treatment (LSVT LOUD) on Swallowing in Patients with Progressive Supranuclear Palsy: A Pilot Study. Prog Rehabil Med. 2021;6:20210012. doi:10.2490/prm.20210012

40. El Sharkawi A, Ramig L, Logemann JA, Pauloski BR, Rademaker AW, Smith CH, Pawlas A, Baum S, Werner C. Swallowing and voice effects of Lee Silverman Voice Treatment (LSVT): a pilot study. J Neurol Neurosurg Psychiatry. 2002;72:31-6. doi 10.1136/jnnp.72.1.31

41. Pauloski BR, Yahnke KM. Using Ultrasound to Document the Effects of Expiratory Muscle Strength Training (EMST) on the Geniohyoid Muscle. Dysphagia. 2021;doi:10.1007/s00455-021-10328-x

42. Kakehi S, Wakabayashi H, Inuma H, Inose T, Shioya M, Aoyama $\mathrm{Y}$, et al. Rehabilitation Nutrition and Exercise Therapy for Sarcopenia. World J Mens Health 2022;40:1-10. doi:10.5534/wjmh.200190

How to cite this article: Wakabayashi, H., Kishima, M., Itoda, M. et al. Prevalence of Hoarseness and Its Association with Severity of Dysphagia in Patients with Sarcopenic Dysphagia. J Nutr Health Aging. 2022;26(3):266-271, https://doi.org/10.1007/s12603 022-1754-3 